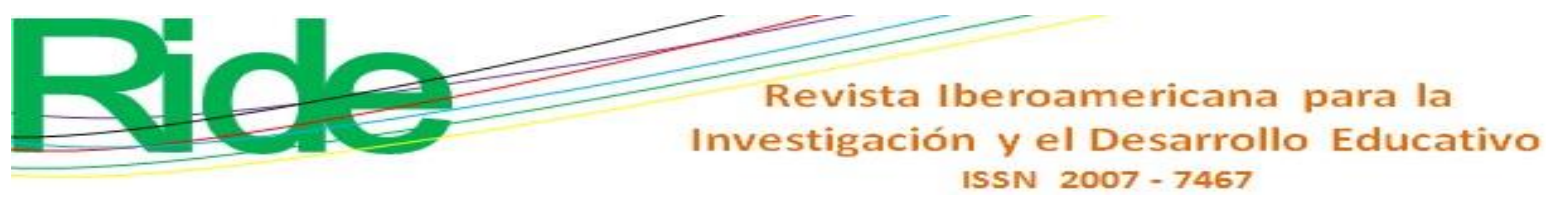

https://doi.org/10.23913/ride.v10i20.662

Artículos Científicos

\title{
El capital estructural y su relación con el desempeño en educación superior
}

\section{Structural capital and its relationship with performance in higher education}

\section{Capital estrutural e sua relação com o desempenho no ensino superior}

Jesús Lavín Verástegui

Universidad Autónoma de Tamaulipas, México

jelavinv@gmail.com

https://orcid.org/0000-0002-8590-664X

\section{Resumen}

El objetivo del presente estudio fue analizar la relación del capital estructural y el desempeño en escuelas de educación superior. Para ello, se llevó a cabo un estudio de naturaleza cuantitativa y se aplicó un cuestionario al personal de instituciones de educación superior. La muestra estuvo conformada por 84 participantes. Mediante la técnica de análisis factorial exploratorio, se identificó la estructura subyacente de los constructos de interés y por medio del coeficiente de correlación se determinó la asociación entre las variables de capital estructural y desempeño. Se determinó que existe una correlación positiva y significativa del capital estructural con las dimensiones del desempeño: calidad educativa y eficacia-innovación. Estos hallazgos son relevantes porque dan pauta a la gestión directiva de las instituciones analizadas sobre la trascendencia de implementar prácticas de diseño y técnicas de organización relacionadas con la estructura formal de las escuelas, lo que brinda beneficios al desempeño organizacional.

Palabras clave: capital intelectual, capital estructural, desempeño organizacional, intangibles, instituciones de educación superior. 


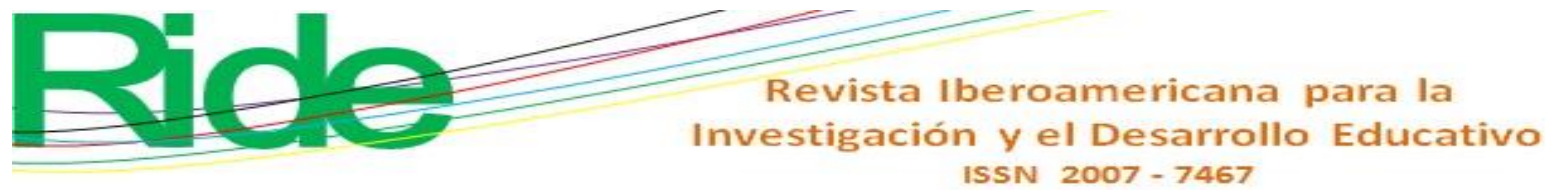

\section{Abstract}

The objective of this study was to analyze the relationship between structural capital and performance in higher education schools. A quantitative study was carried out, for which a questionnaire was applied to personnel of higher education institutions who agreed to answer the instrument. A sample of 84 respondents was obtained. The exploratory factor analysis technique identified the underlying structure of the constructs of interest, and by means of the correlation coefficient, the association between structural capital and performance variables was determined. It was determined that there is a positive and significant correlation of structural capital with the dimensions of performance: educational quality and efficiency-innovation. These findings are relevant because they give guidance to the management of the institutions analyzed, the importance of implementing design practices and implementation of organizational techniques related to the formal structure of schools for the benefits it brings to organizational performance.

Keywords: Intellectual capital, structural capital, organizational performance, intangibles, higher education institutions.

\section{Resumo}

O objetivo do presente estudo foi analisar a relação entre capital estrutural e desempenho em escolas de ensino superior. Para isso, foi realizado um estudo de natureza quantitativa e aplicado um questionário aos funcionários das instituições de ensino superior. A amostra foi composta por 84 participantes. Utilizando a técnica de análise fatorial exploratória, a estrutura subjacente dos constructos de interesse foi identificada e a associação entre o capital estrutural e as variáveis de desempenho foi determinada pelo coeficiente de correlação. Foi determinado que existe uma correlação positiva e significativa do capital estrutural com as dimensões do desempenho: qualidade educacional e efetividade-inovação. Esses achados são relevantes, pois orientam o gerenciamento das instituições analisadas sobre a importância da implementação de práticas de design e técnicas de organização relacionadas à estrutura formal das escolas, o que traz benefícios ao desempenho organizacional.

Palavras-chave: capital intelectual, capital estrutural, desempenho organizacional, intangíveis, instituições de ensino superior.

Fecha Recepción: Octubre 2019

Fecha Aceptación: Mayo 2020 


\section{Introducción: la importancia del desempeño organizacional (DO)}

El desempeño organizacional (DO) es uno de los conceptos más relevantes en la administración, y en diversas ocasiones se le reconoce como sinónimo de productividad o excelencia organizativa (Richard, Devinney, Yip y Johnson, 2009; Wee y Chua, 2015). Tradicionalmente, el DO se asocia a los ingresos y la rentabilidad; sin embargo, de acuerdo con Ousama, Fatima y Hafiz (2011), la incertidumbre económica y la competencia global vuelven insuficiente esta visión para proveer una perspectiva adecuada del presente y futuro, por lo que se han empleado otros elementos de medición, como lo son la continuidad de la organización en el mercado, la gestión de riesgos y la productividad organizacional (Pal, Torstensson y Mattila, 2011).

La medición del desempeño, por tanto, ha adquirido mayor complejidad en las últimas décadas tras la aparición de teorías orientadas a evaluarlo mediante modelos multivariables, en lugar de utilizar únicamente el elemento financiero (Aydogan, 2011; Hornungova, 2014; Vasconcelo, Pedraza, Lavín y Cortés, 2016). De esta forma, la literatura existente muestra la necesidad de contar con indicadores que cubran a la institución de manera holística, y no solo focalizada, ya que el centrar la atención en elementos tradicionales - como el financiero- limita la posibilidad de incluir dentro de la medición aquellos recursos y capacidades desarrolladas por la organización, que han mostrado ser de importancia para la producción y el desempeño de las empresas (Grant, 1991; Wernerfelt, 1984). Asimismo, su adquisición y explotación contribuyen al desempeño superior (Barkat, Beh, Ahmed y Ahmed, 2018).

Numerosos estudios se han enfocado en medir el desempeño con dimensiones que trascienden los elementos tangibles, con la aplicación del capital intelectual (CI) (Asiaei y Jusoh, 2015; Bontis, 1998; Bontis, Chua y Richardson, 2000), y en múltiples casos se ha demostrado que tiene una influencia significativa. Este impacto positivo se muestra en estudios de diversos sectores, como el bancario o el de telecomunicaciones (Cabrita y Bontis, 2008; Latif, Malik y Aslam, 2012; Khalique, Shaari, Isa y Samad, 2013; Mention y Bontis, 2013; Mondal y Ghosh, 2012; Saeed, Sami, Lodhi y Iqbal, 2013; Suraj y Bontis, 2012), donde en general se ha evidenciado una influencia significativa del capital intelectual y sus dimensiones sobre el DO.

En este sentido, uno de los entes que fungen como un área de estudio de relevancia para la medición de su desempeño son las instituciones de educación superior (IES), en su carácter de actores clave de la sociedad del conocimiento debido al rol primordial que tienen en la preparación del capital humano (Galleguillos, Silva y Becerra, 2018). Al respecto, Smart y Hamm (1993) comentan que la medición del desempeño de una universidad es de suma importancia si la 


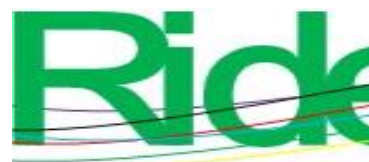

Revista Iberoamericana para la Investigación y el Desarrollo Educativo

ISSN $2007-7467$

institución desea entregar resultados de calidad, ya que es un indicador amplio que puede incluir productividad, calidad, consistencia, etc. En última instancia, el éxito de una institución se evaluará según el desempeño de esta en relación con los objetivos fijados (Richard et al., 2009). No obstante, es necesario identificar cuáles son los principales elementos que contribuyen a ese desempeño, como lo es el caso de los activos intangibles con los que cuenta y que forman su capital intelectual (CI).

\section{La evolución y naturaleza del capital intelectual (CI)}

El capital intelectual, visto como el conocimiento orientado de la organización, ha tomado notable relevancia en los últimos años (Campisi y Costa, 2008). Esta teoría muestra la importancia de la gestión de intangibles dentro de las organizaciones al ser reconocido como el conocimiento que genera valor a la organización mediante su adecuada gestión (Mercado y Cernas, 2012). Integra elementos que ayudan a la organización a alcanzar resultados en el largo plazo Choudhury (2010) como pueden ser patentes, marcas, relaciones con clientes y conocimientos, por lo que se puede establecer que la capacidad real de una institución se encuentra oculta en estos intangibles (Shehzad, Fareed, Zulfiqar, Shahzad y Latif, 2014). En el campo de la educación, por su parte, Ramírez y Gordillo (2014) señalan que el CI integra los activos no tangibles de la institución, como las patentes, derechos de autor, procesos, capacidad de innovación, reconocimiento de la sociedad, capacidades y conocimientos de los miembros, competencias, habilidades y redes.

Tradicionalmente, se ha establecido como modelo de esta teoría el desarrollado por Bontis (1998), quien establece una división del capital intelectual en tres dimensiones de relevancia para cualquier institución:

- Capital humano $(\mathrm{CH})$. Se refiere al conjunto de valores, creencias, capacidades y actitudes que poseen los miembros de la organización, fundamentales para la generación de valor dentro de ella (Bontis et al., 2000; Bueno et al., 2003). Se refleja particularmente en el actuar de las personas, quienes, a partir del proceso enseñanza-aprendizaje, realizan sus labores de manera concreta, además de — con la experiencia adquirida - promover la innovación al ejecutar sus funciones de forma creativa (Brooking, 1997; Edvinsson y Malone, 1999).

- Capital relacional (CR). Hace referencia a todas aquellas interacciones con el exterior que promueven la generación de valor para la organización en general o particularmente para sus productos y servicios (Bontis et al., 2000). Entre los principales agentes que se incluyen 


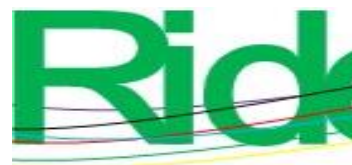

\section{Revista Iberoamericana para la Investigación y el Desarrollo Educativo \\ ISSN $2007-7467$}

en dichas interacciones se pueden mencionar proveedores, competidores y clientes (Martos, Fernandez y Froilan, 2008).

- Capital estructural (CE). Permite identificar aquel conocimiento interno que posee la organización y colabora para la generación de su valor; a diferencia del humano, este tipo de capital permanece aun cuando los trabajadores se marchan de la institución, permitiendo que siga siendo utilizado por nuevos miembros (Roos, Roos, Dragonetti y Edvinsson, 1997). Ejemplos de capital estructural pueden observarse cuando se habla de los procesos dentro de una empresa, la cultura organizacional, los sistemas y tecnologías utilizadas, así como la propia estructura organizacional y el trabajo en equipo (Martos et al., 2008).

Según Marr, Gray y Neely (2003), las razones para medir el CI de una organización radican en que esto permitirá ayudar a la empresa a formular su estrategia, evaluar su ejecución, colaborar en la toma de decisiones, comunicarse de manera efectiva con los agentes de interés y para tomar como base de la compensación de los administradores, es decir, será una noción que sirva para identificar el desempeño que tiene la organización (Todericiu y Serban, 2015).

De acuerdo con Rodríguez, Ortega-Fernández, Alvarez y Roqueñí (2006), medir el capital intelectual de una organización es fundamental para poder realizar comparaciones más realistas de su situación, de manera que pueda ser estimado su valor y controlar su desempeño y crecimiento. Lo anterior es respaldado por Guthrie, Ricceri y Dumay (2012) y por Rodrigues, Selig y Viegas (2019), quienes comentan el cambio de paradigma que desde 1980 la economía mundial ha transitado de una visión industrial a un marco basado en el conocimiento y de ahí la importancia de contar con una medición del CI que permita identificar la creación de valor y de ventajas competitivas. Este enfoque es compartido por Rodrigues, Figueroa y Fernández-Jardón (2009), quienes comentan la importancia del gasto en los activos intangibles, ya que representan variables que impactan de manera significativa en el desempeño de las organizaciones.

\section{Relación del capital intelectual y el desempeño organizacional}

Como lo muestra la literatura existente, el capital intelectual es un tema estudiado de manera amplia en países desarrollados e industrializados, aunque no así en economías emergentes, mucho menos en instituciones de educación superior (IES) (Tseng y Goo, 2005). En México, este hecho es también una realidad, al existir poca investigación al respecto orientada a universidades (Mercado-Salgado, Cernas-Ortiz y Sánchez, 2014). 


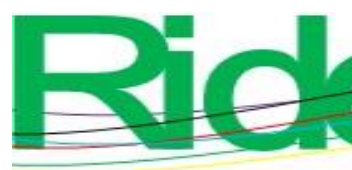

Revista Iberoamericana para la Investigación y el Desarrollo Educativo

ISSN $2007-7467$

Las IES — desde hace más de una década - están inmersas en procesos de cambio de paradigma que buscan crear organizaciones con un mayor nivel de competitividad, dinamismo y transparencia (Sánchez, Elena y Castrillo, 2009). En este sentido, Zorrilla (2010) argumenta que debe implementarse una filosofía de gestión que contribuya a fortalecer las universidades y a mejorar sus resultados de competitividad y credibilidad en la sociedad, esto mediante la gestión del conocimiento; enfatizando en la relación que tiene la gestión del capital intelectual con el desempeño de las instituciones, al describir la relación entre sus dimensiones con el impacto que genera en los procesos que desempeña una IES y, por ende, en los agentes que interactúan con la organización.

Como se comentaba previamente, resulta esencial observar los resultados de la relación entre los componentes del capital intelectual y como estos, en su carácter de variable independiente, pueden ser capaces de impactar en el desempeño organizacional como variable dependiente; no obstante, es necesario identificar en primera instancia si las dimensiones comentadas aplican al contexto educativo. En este sentido, como lo comentan Demuner, Nava e Ibarra (2014), la clasificación descrita es también aplicable a instituciones de educación superior, y existe una serie de estudios que muestran cómo son relevantes para el contexto universitario las interrelaciones entre las tres dimensiones del capital intelectual (Bezhani, 2010; Leitner, 2004; Ramírez, Lorduy y Rojas, 2007; Sánchez et al., 2009).

Por ejemplo, el capital humano se percibe como los conocimientos desarrollados por la comunidad universitaria, así como sus experiencias y competencias, derivadas de la actualización académica y el trabajo en equipo (Bueno et al., 2002; Ramírez et al., 2007); el capital relacional es identificado en las redes de trabajo de alumnos y empresas, ya que estas permiten la transferencia de conocimiento hacia adentro y hacia afuera de la universidad. Por su parte, el capital estructural "integra el conocimiento incorporado, sistematizado y procesado de la universidad, que una vez formalizado llega a ser de su propiedad y se transmite a alumnos, organizaciones y a la sociedad misma" (Demuner, Nava e Ibarra, 2016, p. 64), en otras palabras, es el conocimiento propio de la institución que se sistematiza hasta el grado de generar rutinas (Bueno, 2003). Además, funciona como un medio que permite buscar la rentabilidad y proyección del talento e inteligencia de todos sus miembros, consiguiendo así conformar la propuesta de valor de la organización (Bueno, 2003).

Robles y Zárate (2013) recalcan la importancia de estudiar la forma de gestión del capital intelectual en las universidades, dada la fuente de valor que esto representaría para la institución. En este orden de ideas, existen casos de investigación sobre la relación entre las variables del CI y 


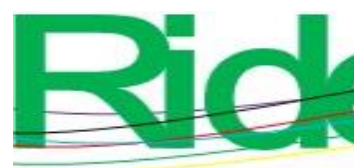

Revista Iberoamericana para la Investigación y el Desarrollo Educativo

ISSN $2007-7467$

el DO en el contexto educativo, como se puede observar en el estudio de Shehzad et al. (2014), quienes lo abordan desde el contexto universitario de Pakistán. Sus resultados muestran una correlación positiva entre las tres dimensiones del CI con el desempeño institucional de las universidades pakistaníes, lo que muestra la enorme contribución que se tiene entre estas variables.

Otros estudios, como el de Galleguillos et al. (2018), enseñan también una relación positiva entre las tres dimensiones del CI y el desempeño de las universidades; no obstante, recalcan que solo algunos indicadores de cada dimensión son los que aportan significancia a esta correlación. Este hecho se da en el contexto de las instituciones de educación superior de Chile, orientando el desempeño exclusivamente al punto de vista financiero, y se señalan como principales indicadores que llevan a la mejora el orientado al incremento de académicos de tiempo completo, las acreditaciones, la generación de proyectos apoyados por el gobierno y las publicaciones.

Bermúdez, Pertuz, y Boscan (2015) establecen una visión del capital estructural en universidades de Colombia desde una perspectiva cualitativa, al identificar fortalezas y debilidades en ese contexto. Concluyen que los elementos positivos se orientan a esfuerzos de investigación y desarrollo y dotación tecnológica, mientras que las debilidades se enfocan hacia aspectos de propiedad intelectual y de innovación. Por su parte, Merhej y Deeb (2016) hablan del impacto que tiene el capital estructural en el desempeño innovativo en universidades de Siria, y logran establecer una correlación significativa entre estas dos variables. Sin embargo, mencionan que esta relación es débil debido al retraso existente en los campos de las tecnologías de la información donde se encontró que los sistemas utilizados en aquel país no son efectivos.

La literatura muestra también estudios desarrollados a partir de cada una de las dimensiones del CI para comprobar la relación existente con el DO. Un ejemplo de ello se puede observar en el estudio de Barkat et al. (2018), quienes buscan la relación entre el capital humano, capital estructural y capital relacional con el desempeño organizacional en empresas textiles de Pakistán. En este caso, se encontró que dos de las tres dimensiones tenían una relación significativa con el desempeño organizacional, siendo el capital relacional el que cuenta con el efecto más fuerte, dejando al capital humano en segundo lugar. En ese estudio, además, se muestran efectos insignificantes entre el capital estructural y el DO.

El estudio de Barkat et al. (2018) es consistente con el de Hormiga, Batista-Canino y Sánchez-Medina (2011), quienes comentan que una parte significativa del desempeño organizacional está dado por la relación entre la empresa con los clientes y proveedores en el contexto de los startups de España y Portugal. De forma similar Luo, Griffith, Liu y Shi (2004) 


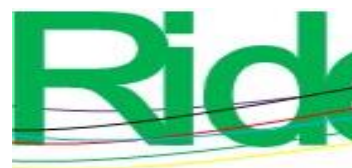

Revista Iberoamericana para la Investigación y el Desarrollo Educativo

ISSN $2007-7467$

demuestran una relación significativa entre el CR y el DO, principalmente hacia el desempeño estratégico y financiero. Efectos positivos entre CH y DO también se hallan en estudios de Wang, Wang y Liang (2014) y Felicio, Couto y Caiado (2014), quienes destacan el rol de la correcta gestión del recurso humano para conseguir el éxito dentro de las organizaciones.

Por otra parte, sin embargo, se pueden apuntar relaciones insignificantes entre el CE y el DO. Por ejemplo, Leitner (2015) menciona que existe un efecto negativo entre estas variables en el largo plazo, principalmente sobre la rentabilidad y el crecimiento de la empresa, al menos en el contexto de Austria. Asimismo, Hejazi, Ghanbari y Alipour (2016) explican que el capital estructural tiene una relación negativa significativa con el desempeño de una empresa, estableciendo en ese estudio que la principal dimensión que impacta en el DO es el capital humano.

Sin embargo, estudios como el de Hsu y Wang (2012) muestran que el capital estructural está asociado de manera positiva con el desempeño de una organización. Esto también es apoyado por Al-Hawajreh (2013), quien encuentra una relación positiva entre el capital estructural y el desempeño organizacional en el sector farmacéutico de Jordania; además, muestra que si bien la variable de CE completa tiene una relación significativa, no todos sus indicadores tienen el mismo peso.

Gogan, Artene, Sarca y Draghici (2016), por su parte, publican resultados positivos en las tres dimensiones del CI y su relación con el DO. En el capital humano evidencian una correlación superior a 0.5, y determinan un rol dominante del $\mathrm{CH}$ sobre el desempeño organizacional. En cuanto al capital estructural, a pesar de que en una de las cuatro empresas analizadas la relación es negativa, en las otras tres se evidencia una asociación positiva, lo que muestra una influencia significativa sobre el DO. Finalmente, el CR también fue encontrado con relación significativa positiva, por lo que en el marco de la revisión de literatura expuesta en la presente discusión se establece la siguiente hipótesis general de investigación (HGI): desde la percepción de los encuestados, existe una correlación positiva y significativa entre el capital estructural y el desempeño organizacional en las instituciones de educación superior analizadas en el presente estudio. 


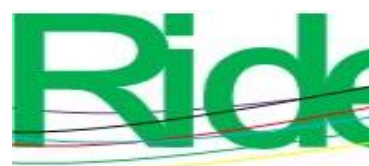

Revista Iberoamericana para la Investigación y el Desarrollo Educativo

ISSN $2007-7467$

\section{Material y método}

La presente investigación se sustentó en el paradigma cuantitativo con la intención de valorar, desde la percepción de los encuestados, la importancia que otorgan a la relación de los aspectos de capital estructural con el desempeño. Para lograr este cometido se diseñó un cuestionario con una escala tipo Likert de cinco puntos, la cual se enseñó a los docentes y directivos participantes. Para la variable de capital estructural el número 1 representaba nunca y 5 siempre, mientras que para la variable de desempeño el número 1 significaba pésimo y 5 superior. Cabe señalar que el diseño del cuestionario tomó como referencia general el modelo propuesto por Bontis (1998), quien hace una propuesta de la operacionalización del capital intelectual, el cual —en el caso concreto de la presente investigación - se tradujo y adoptó para el factor del capital estructural, mientras que para la variable DO se propusieron indicadores considerados propios para evaluar a la universidad.

El cuestionario quedó constituido por tres apartados: el primero estuvo conformado por 15 reactivos para recoger la valoración de los encuestados sobre el capital estructural; la segunda sección se diseñó con 14 preguntas de indicadores referentes al desempeño organizacional de las universidades; finalmente, en la última sección se plantearon preguntas para recabar datos referentes a los encuestados y a sus instituciones (p. ej., el cargo desempeñado, la antigüedad en el puesto, la edad del encuestado, el grado de escolaridad y el sexo, mientras que en relación con la organización se procuró conocer si correspondía al sector público o privado, la ubicación —norte, centro, sur - y años de servicio de la institución en la sociedad).

Se logró conformar una muestra a conveniencia de 84 encuestados de instituciones públicas y privadas que decidieron colaborar en la investigación. La recolección de los datos se realizó entre marzo y junio de 2019. El contacto con los directivos de las IES se estableció por medio de oficio, en el cual se expuso el objetivo del estudio y la invitación a colaborar. En ese primer acercamiento formal se aplicó el cuestionario de forma presencial al personal directivo y docente que accedió a responder el instrumento. Para presentar las características de los encuestados, se acudió a la estadística descriptiva, que implica resumir los datos resultantes de un trabajo de investigación de manera que puedan ser compilados, organizados, analizados y expuestos por medio de elementos que los muestren de manera clara y sencilla, como tablas, cuadros y gráficas, entre otros (López y Diez, 2017; Rendón-Macías, Villasís-Keever y Miranda-Novales, 2016). 


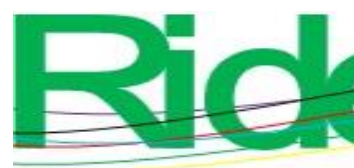

Revista Iberoamericana para la Investigación y el Desarrollo Educativo

ISSN $2007-7467$

La muestra del estudio se caracterizó por lo siguiente: $45.2 \%$ hombres y $54.8 \%$ mujeres. La mitad ocupa el puesto de docente, mientras que la otra realiza actividades de gestión administrativa y académica; esto significa que se consiguió una participación equilibrada de ambos cargos en la organización. La mayoría (60 \%) tenía más de cinco años de antigüedad en la IES y solo $40 \%$ menos de ese tiempo. Respecto a la edad de los encuestados, se identificó que un porcentaje bajo (8.3 \%) tenía entre 18 y 33 años, mientras que la mayoría tenía más de 34 años de edad $(91.70 \%)$.

Una vez conformada la base de datos de los encuestados, mediante el software estadístico SPSS (versión 25) se procedió a aplicar las técnicas de análisis de datos —en concreto el análisis factorial exploratorio (AFE) - con el propósito de identificar y constatar la estructura subyacente de las dos variables de estudio: capital estructural y desempeño organizacional. Esta técnica de análisis de datos consiste en "un conjunto de métodos estadísticos multivariados de interdependencia cuyo propósito principal es el de identificar una estructura de factores subyacentes a un conjunto amplio de datos" (Pérez y Medrano, 2010, p. 58).

Cabe señalar que la confiabilidad de las escalas se valoró mediante el índice alfa de Cronbach, que establece un valor mínimo de 0.70 para mostrar una adecuada consistencia interna del instrumento. A pesar de que existen distintos métodos, el alfa de Cronbach resulta ser el más utilizado para la estimación de la confiabilidad, siendo el intervalo entre 0.70 y 0.90 el resultado que muestra una buena consistencia interna para una escala unidimensional (Celina y Campo, 2005). Posteriormente, para evaluar la hipótesis de investigación se determinó el coeficiente de correlación, herramienta que permite identificar las asociaciones encontradas entre dos variables (Bottasso, 2009).

\section{Resultados del análisis de la correlación del CE y el DO}

En el estudio se determinó que el capital estructural muestra una sola dimensión en su conformación (tabla 1). Dentro de los aspectos valorados desde la percepción de los encuestados, destacan en este constructo la importancia de que las instituciones educativas cuenten con programas de reclutamiento y selección, con estrategias de innovación y vigilancia tecnológica, así como procesos documentados para asegurar la ejecución de las tareas en la organización. Asimismo, se destaca el contar con bases de datos y sistemas de información, y disponer de una estructura organizacional bien definida (organigramas, manuales de organización y procedimientos). 


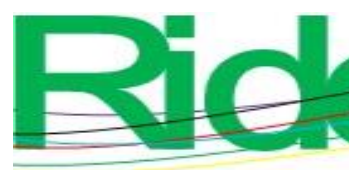

Revista Iberoamericana para la Investigación y el Desarrollo Educativo

ISSN $2007-7467$

La conformación de un solo factor resultó adecuada, pues mostró un nivel de consistencia interna favorable (alfa de 0.85); lo mismo sucedió con la técnica de análisis factorial exploratorio (AFE), que arrojó un valor de KMO superior a 0.70. Cabe señalar que la escala original que se presentó a los encuestados disponía de 15 reactivos para medir el capital estructural; sin embargo, fueron eliminados nueve. debido a su baja carga factorial, de modo que quedaron solo seis indicadores de este constructo, los cuales se observan a detalle en la tabla 1.

Tabla 1. Validación del constructo de capital estructural (CE)

\begin{tabular}{|c|c|c|c|c|}
\hline Factor: Capital estructural & $\begin{array}{l}\text { Media del } \\
\text { ítem }\end{array}$ & $\begin{array}{c}\text { Carga } \\
\text { factorial }\end{array}$ & AFE & $\begin{array}{l}\text { Alfa de } \\
\text { Cronbach }\end{array}$ \\
\hline $\begin{array}{l}\text { CE2 Los programas de reclutamiento y } \\
\text { selección están dedicados para contratar a los } \\
\text { mejores candidatos. }\end{array}$ & 3.69 & 0.81 & \multirow{6}{*}{$\begin{array}{l}\text { KMO } 0.79 \\
\text { Chi } 222.380 \\
\text { Sig. } 0.000\end{array}$} & \multirow{6}{*}{0.85} \\
\hline $\begin{array}{l}\text { CE3 Se desarrollan de forma exitosa programas } \\
\text { de sucesión de puestos. }\end{array}$ & 3.35 & 0.78 & & \\
\hline $\begin{array}{l}\text { CE4 Se cuenta con estrategias de innovación y } \\
\text { vigilancia tecnológica. }\end{array}$ & 3.57 & 0.67 & & \\
\hline $\begin{array}{l}\text { CE10 Se tiene procedimientos documentados } \\
\text { que ayudan a ejecutar acciones rutinarias. }\end{array}$ & 3.98 & 0.78 & & \\
\hline $\begin{array}{l}\text { CE14 Se cuenta con sistemas de información } \\
\text { computarizados. }\end{array}$ & 4.13 & 0.76 & & \\
\hline $\begin{array}{l}\text { CE15 Se cuenta con una estructura } \\
\text { organizacional definida. }\end{array}$ & 4.3 & 0.72 & & \\
\hline
\end{tabular}

Fuente: Elaboración propia

Al correr la técnica de AFE para la variable del desempeño organizacional, se identificaron dos factores, que por las agrupaciones de las variables se denominaron calidad académica (CA) y eficacia e innovación (EI) (tabla 2). Se determinó que el nivel de confiablidad de esta escala también fue aceptable, puesto que el alfa de Cronbach estuvo en el rango aceptable (superior a 0.70), valor obtenido en ambos componentes del constructo de desempeño organizacional en las instituciones educativas analizadas. Se precisa que cuatro reactivos fueron eliminados por las bajas cargas factoriales recibidas, quedando finalmente los indicadores para medir el desempeño en diez reactivos que se distribuyeron en los dos factores descritos en la tabla 2. 


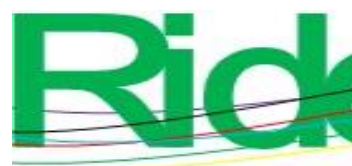

Revista Iberoamericana para la Investigación y el Desarrollo Educativo

ISSN $2007-7467$

Tabla 2. Valoración de la confiabilidad y estructura subyacente del desempeño organizacional

\begin{tabular}{|c|c|c|c|c|}
\hline Factor 1: Calidad Académica (CA) & $\begin{array}{l}\text { Media del } \\
\text { ítem }\end{array}$ & $\begin{array}{l}\text { Carga } \\
\text { factorial }\end{array}$ & AFE & $\begin{array}{l}\text { Alfa de } \\
\text { Cronbach }\end{array}$ \\
\hline D1 De la imagen de su institución y servicios. & 4.27 & 0.74 & \multirow{11}{*}{$\begin{array}{c}\text { KMO } 0.91 \\
\text { Chi } 602.331 \\
\text { Sig. } 0.000\end{array}$} & \multirow{6}{*}{0.89} \\
\hline $\begin{array}{l}\text { D2 De la calidad académica de los procesos y } \\
\text { servicios. }\end{array}$ & 4.35 & 0.85 & & \\
\hline D3 De la formación de calidad de estudiantes. & 4.24 & 0.84 & & \\
\hline D5 De la satisfacción de los usuarios. & 4.11 & 0.72 & & \\
\hline D6 Del desempeño en el logro de objetivos. & 4.17 & 0.69 & & \\
\hline D9 De la productividad de empleados. & 3.98 & 0.75 & & \\
\hline Factor 2: Eficacia e innovación (EI) & & & & \\
\hline D11 Desempeño en aplicación de recursos. & 3.99 & 0.81 & & \multirow{4}{*}{0.91} \\
\hline D12 Coordinación de procesos internos. & 3.94 & 0.75 & & \\
\hline D13 Innovación continua. & 3.99 & 0.77 & & \\
\hline $\begin{array}{l}\text { D14 Vinculación con el sector productivo y } \\
\text { sociedad. }\end{array}$ & 4.08 & 0.86 & & \\
\hline
\end{tabular}

Fuente: Elaboración propia

Posteriormente, una vez identificada la estructura subyacente con la técnica del AFE para el capital estructural, así como para la variable de desempeño organizacional se procedió a evaluar el nivel de correlación entre las variables de interés. De este modo, se confirmó que el capital estructural se correlaciona positiva y significativamente con los dos factores determinados del desempeño en las IES analizadas, con valores positivos y significativos de correlación, que encontraron de 0.48 a 0.53 , como se observa en la tabla 3 , de ahí que no se rechace la hipótesis de investigación del presente estudio. La evidencia empírica de este estudio, por tanto, aporta a la teoría de que los intangibles se asocian con el desempeño de las organizaciones, con lo que se confirma ese supuesto también para el contexto de la educación superior. 


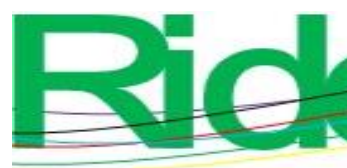

Revista Iberoamericana para la

Investigación y el Desarrollo Educativo

ISSN $2007-7467$

Tabla 3. La correlación entre las variables de estudio

\begin{tabular}{|l|l|c|c|}
\hline Matriz & & $\begin{array}{l}\text { Calidad } \\
\text { académica (CA) }\end{array}$ & $\begin{array}{l}\text { Eficacia e } \\
\text { innovación (EI) }\end{array}$ \\
\hline $\begin{array}{l}\text { Coeficiente de } \\
\text { correlación de } \\
\text { Spearman }\end{array}$ & $\begin{array}{l}\text { Capital } \\
\text { estructural }\end{array}$ & .486 & \\
\hline & $\begin{array}{l}\text { Capital } \\
\text { estructural }\end{array}$ & 0.000 & .531 \\
\hline Significancia & & 84 & 0.000 \\
\hline \multicolumn{1}{|c|}{} & & & 84 \\
\hline
\end{tabular}

Fuente: Elaboración propia

Nota: $\mathrm{N}=$ número de elementos de la muestra de datos.

\section{Discusión, limitaciones e implicaciones del estudio}

Estudios previos de organizaciones tanto en el contexto de las universidades como en otros tipos de industrias han mostrado que existen relaciones positivas y significativas entre los componentes del CI y el DO, resultados que han sido determinados en investigaciones como las realizadas por Al-Hawajreh (2013), Barkat et al. (2018), Galleguillos et al. (2018), Gogan et al. (2016), Merhej y Deeb (2016), Shehzad et al. (2014), Hsu y Wang (2012).

En ese sentido, los hallazgos del presente estudio muestran altos y significativos coeficientes de correlación entre el capital estructural y la calidad académica (coeficiente: 0.48) y la eficacia e innovación (coeficiente: 0.531); sin duda, estos datos aportan evidencia empírica para confirmar los resultados determinados por los estudios referidos anteriormente, que precisamente señalan haber encontrado asociaciones positivas entre estas dos variables (CE y DO).

Cabe precisar que, a la vez, los resultados de este estudio contrastan con los expuestos por Hejazi et al. (2016), quienes señalan que el capital estructural tiene una relación negativa con el desempeño organizacional. Estos autores, de hecho, demuestran en su estudio que la principal dimensión que impacta en el DO es el capital humano. Por ende, se estima que una futura línea de investigación a seguir sería incorporar la dimensión del capital humano como una variable más para analizar, puesto que finalmente son los empleados de una organización quienes poseen activos propios (experiencia, conocimiento, actitud, valores). En otras palabras, cuando ellos se van de la organización, se llevan esos activos intangibles. 


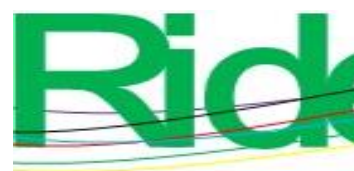

Revista Iberoamericana para la Investigación y el Desarrollo Educativo

ISSN $2007-7467$

En la literatura se señala que el desempeño organizacional tiende a considerarse como sinónimo de productividad (Richard et al., 2009; Wee y Chua, 2015), y en este estudio se confirma este supuesto, porque en la presente investigación dicha variable muestra una estructura factorial determinada por dos factores, identificados como calidad académica (CA) y eficacia e innovación (EI), ambos conformados por aspectos que necesariamente refieren a indicadores relacionados con temas de productividad, como calidad de los procesos, calidad de los servicios, calidad en la formación, logro de objetivos y eficacia.

Por ende, y para buscar cierta generalización de los resultados del presente estudio, es necesario pensar en la posibilidad de replicar la investigación en otros estados del país, pues de ese modo se pueden observar las posibles tendencias en las IES tanto públicas como privadas de México, así como asegurar una muestra representativa de este tipo de organizaciones educativas. En caso contrario, los hallazgos de este estudio tendrían la principal limitación y debilidad de haber sido recabados solo en las instituciones de Tamaulipas que colaboraron en la investigación. Aun así, vale acotar que estos resultados muestran un alcance exploratorio que permite conocer el fenómeno analizado desde la percepción de los docentes y directivos encuestados.

\section{Conclusiones}

Como se ha observado en la literatura comentada, el capital intelectual es un fenómeno estudiado ampliamente en países desarrollados e industrializados, aunque no de la misma manera en las economías emergentes, como es el caso de México. De allí el interés por llevar a cabo investigaciones en nuestro contexto, pues hoy en día las organizaciones, tanto públicas como privadas, se hallan en una búsqueda exhaustiva de estrategias para mantenerse vigentes en sus mercados.

Por ende, el objetivo del presente estudio ha sido analizar de qué manera los aspectos relacionados con las estructuras, los procesos, los programas, los procedimientos y las políticas organizacionales (capital estructural) son valorados por los actores de las IES (directivos y docentes), y cómo ellos aprecian que dichos elementos puedan estar correlacionados con los diferentes desempeños de las instituciones. En ese sentido, el principal hallazgo de esta indagación ha sido confirmar la hipótesis planteada, es decir, que aspectos intangibles —en particular, los agrupados en la categoría de capital estructural - efectivamente muestran correlaciones positivas y significativas con los dos tipos de desempeño determinados en esta investigación: calidad académica (CA) y eficacia e innovación (EI). 


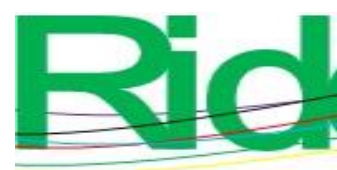

\section{Revista Iberoamericana para la Investigación y el Desarrollo Educativo \\ ISSN $2007-7467$}

Estos resultados son un punto de partida para que la gerencia educativa otorgue importancia a los aspectos formales de su organización no solo para documentar el conocimiento y la experiencia de sus empleados en manuales administrativos, bases de datos y sistemas de información, sino también para estimar cómo pueden beneficiar estrategias de sistemas y procedimientos formales, con el fin de mejorar los indicadores y el logro de objetivos de las organizaciones en materia de calidad educativa, así como procesos y servicios que proporcionan a sus alumnos y a la sociedad en general.

Finalmente, es preciso que los directivos de las IES tomen en cuenta que desde la apreciación de los encuestados se observan dos dimensiones del desempeño que son valoradas de forma importante, por lo que se recomienda a ellos trazar iniciativas de acción que promuevan y fortalezcan los aspectos relativos a dar valor agregado mediante técnicas de organización (capital estructural) que fortalezcan indicadores como la imagen institucional, la calidad en la formación de estudiantes, la calidad de los procesos y servicios educativos, el logro de indicadores académicos, entre otros aspectos valorados positivamente por los encuestados. 


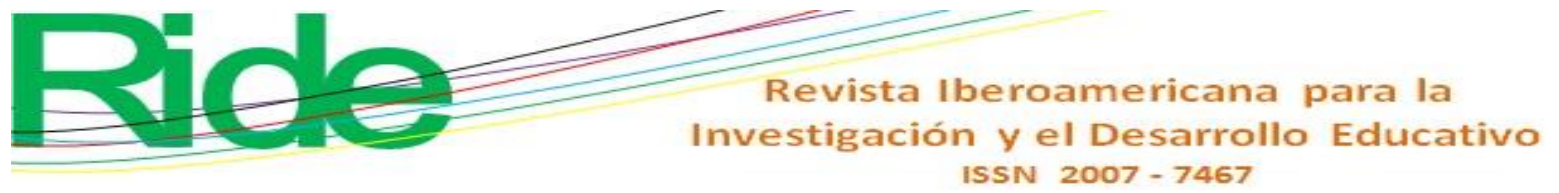

\section{Referencias}

Al-Hawajreh, K. M. (2013). The Impact of Structural Capital on Business Performance in Jordanian Pharmaceutical Manufacturing Companies. European Journal of Business and Management, $\quad 5(10), \quad 2222-2839 . \quad$ Retrieved from https://pdfs.semanticscholar.org/cb0c/d4a68a8902dc8f9e684bd779aa17af40ad3d.pdf

Asiaei , K. and Jusoh, R. (2015). A multidimensional view of intellectual capital: the impact on organizational performance. Management Decision, 53(3), 668-697. Doi: https://doi.org/10.1108/MD-05-2014-0300

Aydogan, E. K. (2011). Performance measurement model for Turkish aviation firms using the rough-AHP and TOPSIS methods under fuzzy environment. Expert Systems with Applications, 38(4), 3992-3998. Doi: http://doi.org/10.1016/j.eswa.2010.09.060

Barkat, W., Beh, L.-S., Ahmed, A. y Ahmed, R. (2018). Impact of intellectual capital on innovation capability and organizational performance: An empirical investigation. Serbian Journal of Management, 13(2), 365-379. Doi: 10.5937/sjm13-16997

Bermúdez, M., Pertuz, V. y Boscan, N. (2015). Capital estructural: análisis diagnóstico en grupos de investigación y desarrollo (I+D) de universidades públicas de Colombia. Revista Universo Contábil, 11(3), 132-149. Recuperado de http://www.redalyc.org/articulo.oa?id=117042808008

Bezhani, I. (2010). Intellectual capital reporting at UK universities. Journal of Intellectual Capital, 11(2), 179-207. Doi: https://doi.org/10.1108/14691931011039679

Bontis, N. (1998). Intellectual capital: an exploratory study develops measure and models. Management Decision, 36(2), 63-76. Doi: https://doi.org/10.1108/00251749810204142

Bontis, N., Chua, W. and Richardson, S. (2000). Intellectual capital and business performance in Malaysian industries. Journal of Intellectual Capital, 1(1), 85-100. Doi: https://doi.org/10.1108/14691930010324188

Bottasso, O. (2009). El coeficiente de correlación, una historia de debates movilizantes. Revista Médica de Rosario, 75, 80-82. Recuperado de http://search.ebscohost.com/login.aspx $?$ direct=true $\& d b=1$ th $\& A N=52428388 \&$ site=ehostlive

Brooking, A. (1997). El capital intelectual. El principal activo de las empresas del tercer milenio. Barcelona: Paidós Ibérica. 


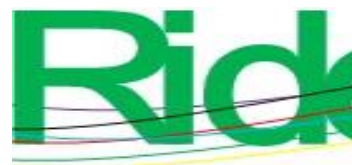

Revista Iberoamericana para la

Investigación y el Desarrollo Educativo

ISSN $2007-7467$

Bueno, E. (2003). Modelo Intellectus: Modelo de medición y gestión del capital intelectual. Madrid: CIC.

Bueno, E., Morcillo, P., Rodríguez, J., Luque, M. Á., Cervera, M., Camacho, C. y Villar, L. (2003). Gestión del Conocimiento en Universidades y Organismos Públicos de Investigación. Madrid: Dirección General de Investigación, Consejería de Educación, Comunidad de Madrid. Recuperado de https://www.madrimasd.org/uploads/informacionidi/biblioteca/publicacion/doc/16_Gestio nConocimientoUniversidadesOPIS.pdf

Bueno, E., Morcillo, P., Rodríguez, J., Luque, M. Á., Cervera, M., Rodríguez, Ó. y Martínez, J. (2002). Indicadores de capital intelectual aplicados a la actividad investigadora y de gestión del conocimiento en las universidades y centros públicos de investigación de la comunidad de Madrid. En Modrego, A. Capital intelectual y producción científica (pp. 19-70). Madrid: Dirección General de Investigación, Consejería de Educación, Comunidad de Madrid. Recuperado de https://www.madrimasd.org/uploads/informacionidi/biblioteca/publicacion/doc/Libro_12c ompleto.pdf

Cabrita, M. and Bontis, N. (2008). Intellectual capital and business performance in the Portuguese banking industry. Int. J. Technology Management, 43(1-3), 212-237. Doi: 10.1504/IJTM.2008.019416

Campisi, D. and Costa, R. (2008). A DEA based method to enhance intellectual capital management. Knowledge and Process Management, 15(3), 170-183. Doi: $10.1002 / \mathrm{kpm} .312$

Celina, H. y Campo, A. (2005). Aproximación al uso del coeficiente alfa de Cronbach. Revista Colombiana de Psiquiatría, 34(4), 572-580. Recuperado de http://www.redalyc.org/articulo.oa?id=80634409

Choudhury, J. (2010). Performance Impact of Intellectual Capital: A Study of Indian it Sector. International Journal of Business and Management, 5(9), 72-80. Doi: 10.5539/ijbm.v5n9p72

Demuner, M., Nava, R. e Ibarra, M. (2014). El capital estructural y sus componentes en las instituciones de educación superior. Una exploración conceptual. XIX Congreso Internacional de Investigación en Ciencias Administrativas. Durango, México. Recuperado de 


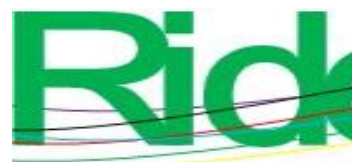

Revista Iberoamericana para la Investigación y el Desarrollo Educativo ISSN $2007-7467$

http://acacia.org.mx/busqueda/pdf/EL_CAPITAL_ESTRUCTURAL_Y_SUS_COMPON ENTES_EN_LAS_INSTITUCIONES_DE_EDUCACION_SUPERIOR._UNA_EXPLO RACION-1.pdf

Demuner, M., Nava, R. e Ibarra, M. (2016). Dimensiones e indicadores de capital estructural para la universidad pública. En Nava, R. M., Mercado, P. y Demuner, M., El capital intelectual en la universidad pública (pp. 61-80). Toluca, México: Universidad Autónoma de Estado de México.

Recuperado de http://ri.uaemex.mx/bitstream/handle/20.500.11799/66109/LibroCIenUP.pdf?sequence=1 \&isAllowed=y

Edvinsson, L. y Malone, M. (1999). El capital intelectual (1. a ed.). Barcelona: Gestión 2000.

Felicio, J. A., Couto, E. and Caiado, J. (2014). Human capital, social capital and organizational performance. Management Decision, 52(2), 350-364. Doi: 10.1108/MD-04-2013-0260

Galleguillos, C., Silva, J. L. y Becerra, P. (2018). Incidencia del capital intelectual en el rendimiento financiero de las Universidades de Chile. Ingeniare. Revista Chilena de Ingeniería, 26(4), 593-598. Doi: 10.4067/S0718-33052018000400593

Gogan, L. M., Artene, A., Sarca, I. y Draghici, A. (2016). The Impact of Intellectual Capital on Organizational Performance. Procedia - Social and Behavioral Sciences, 221, 194-202. Retrieved from https://cyberleninka.org/article/n/640757.pdf

Grant, R. (1991). The resource-based theory of competitive advantage: implications for strategy formulation. California Management Review, 33(3), 114-135. Retrieved from http://www.ftms.edu.my/images/Document/MOD001074\%20\%20Strategic\%20Management\%20Analysis/WK6_SR_MOD001074_Grant_1991.pdf

Guthrie, J., Ricceri, F. y Dumay, J. (2012). Reflections and projections: A decade of Intellectual Capital Accounting Research. The British Accounting Review, 44(2), 68-82. Doi: https://doi.org/10.1016/j.bar.2012.03.004

Hejazi, R., Ghanbari, M. and Alipour, M. (2016). Intellectual, Human and Structural Capital Effects on Firm Performance as Measured by Tobin's Q. Knowledge and Process Management, 23(4), 259-273. Doi: 10.1002/kpm.1529

Hormiga, E., Batista-Canino, R. M. and Sánchez-Medina, A. (2011). The Impact of Relational Capital on the Success of New Business Start Ups. Journal of Small Business Management, 49(4), 617-638. Doi: https://doi.org/10.1111/j.1540-627X.2011.00339.x 


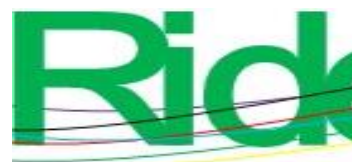

Revista Iberoamericana para la Investigación y el Desarrollo Educativo

ISSN $2007-7467$

Hornungova, J. (2014). Development of concepts and models of performance evaluation fron the 19th century to the present. Danube: Law and Economics Review, 5(2), 143-154. doi:http://doi.org/10.2478/danb-2014-0008

Hsu, L.-C. y Wang, C.-H. (2012). Clarifying the Effect of Intellectual Capital on Performance: The Mediating Role of Dynamic Capability. British Journal of Management, 23(2), 179-205. Doi: 10.1111/j.1467-8551.2010.00718.x

Khalique, M., Shaari, J. A., Isa, A. H. and Samad, N. B. (2013). Impact of Intellectual Capital on the Organizational Performance of Islamic Banking Sector in Malaysia. Asian Journal of Finance \& Accounting, 5(2), 75-83. Doi: 10.5296/ajfa.v5i2.4005

Latif, M., Malik, M. S. and Aslam, S. (2012). Intellectual capital efficiency and corporate performance in developing countries: A comparison between islamic and conventional banks of Pakistan. Interdisciplinary Journal of Contemporary Research in Business, 4(1), 405-420. Retrieved from https://www.researchgate.net/publication/261031515

Leitner, K.-H. (2004). Intellectual capital reporting for universities: conceptual background and applications for Austrian universities. Research evaluations, 13(2), 129-140. Doi: $10.3152 / 147154404781776464$

Leitner, K.-H. (2015). Intellectual Capital, Innovation, and Performance: Empirical Evidence from SMEs. International Journal of Innovation Management, 19(5). Doi: $10.1142 / \mathrm{S} 1363919615500607$

López, A. y Diez, T. (2017). Aproximación de la estadística a las ciencias sociales: una mirada crítica. Revista Cubana Educación Superior, 36(2), 148-156. Recuperado de http://search.ebscohost.com/login.aspx?direct=true \&db=zbh\&AN=126117222\&site=ehos t-live

Luo, X., Griffith, D. A., Liu, S. S. and Shi, Y.-Z. (2004). The Effects of Customer Relationships and Social Capital on Firm Performance: A Chinese Business Illustration. Journal of International Marketing, 12(4), 25-45. Doi: 10.1509/jimk.12.4.25.53216

Marr, B., Gray, D. and Neely, A. (2003). Why Do Firms Measure Their Intellectual Capital? Journal of Intellectual Capital, 4(4), 441-464. Doi: 10.1108/14691930310504509

Martos, M. S., Fernández, C. M. y Froilan, P. (2008). Evaluación y relaciones entre las dimensiones del capital intelectual: El caso de la cadena de la madera de Oberá (Argentina). Intangible Capital, 4(2), 67-101. Recuperado de http://www.redalyc.org/articulo.oa?id=54940201 


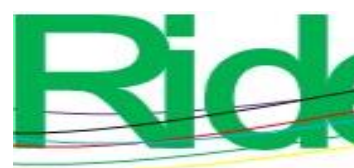

Revista Iberoamericana para la Investigación y el Desarrollo Educativo

ISSN $2007-7467$

Mention, A. L. and Bontis, N. (2013). Intellectual capital and performance within the banking sector of Luxembourg and Belgium. Journal of Intellectual Capital, 14(2), 286-309. Doi: https://doi.org/10.1108/14691931311323896

Mercado, P. y Cernas, D. (2012). El capital intelectual en las organizaciones. Una agenda de investigación. Toluca: Universidad Autónoma del Estado de México.

Mercado-Salgado, P., Cernas-Ortiz, D. A. y Sánchez, M. L. (2014). El capital intelectual en universidades públicas mexicanas. Un estudio comparativo. The Academic Journal of the Universidad Anáhuac, 14, 37-56. Doi: 10.2139/ssrn.2550028

Merhej, M. M. and Deeb, K. A. (2016). The Impact of Structural Capital on the Innovation Performance in Syrian Universities: A Field Study in Tishreen University. European Online Journal of Natural and Social Sciences, 5(3), 664-673. Retrieved from https://www.researchgate.net/profile/Maher_Merhej/publication/305650161_The_Impact _of_Structural_Capital_on_the_Innovation_Performance_in_Syrian_Universities_A_Fiel d_Study_in_Tishreen_University/links/59f35e4b458515547c205378/The-Impact-ofStructural-Capi

Mondal, A. and Ghosh, S. K. (2012). Intellectual capital and financial performance of Indian banks. Journal of Intellectual Capital, 13(4), 515-530. Doi: https://doi.org/10.1108/14691931211276115

Ousama, A., Fatima, A. y Hafiz, A. (2011). Usefulness of intellectual capital information: preparers' and users' views. Journal of Intellectual Capital, 12(3), 430-445. Doi: https://doi.org/10.1108/14691931111154724

Pal, R., Torstensson, H. and Mattila, H. (2011). Organizational resilience and health of business systems. International Journal of Business Continuity and Risk Management, 2(4), 372398. Doi: 10.1504/IJBCRM.2011.044410

Pérez, E. y Medrano, L. (2010). Análisis factorial exploratorio: bases conceptuales y metodológicas. Revista Argentina de Ciencias del Comportamiento, 2(1), 58-66. Recuperado de https://dialnet.unirioja.es/servlet/articulo?codigo=3161108

Ramírez, Y. and Gordillo, S. (214). Recognition and measurement of intellectual capital in Spanish universities. Journal of Intellectual Capital, 15(1), 173-188. Doi: https://doi.org/10.1108/JIC-05-2013-0058 


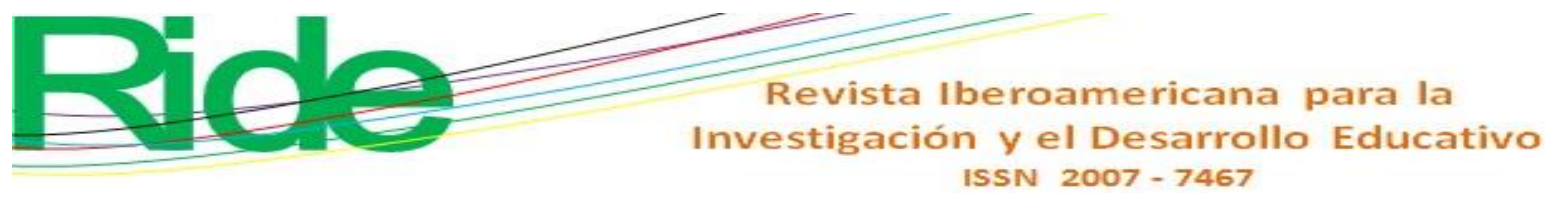

Ramírez, Y., Lorduy, C. and Rojas, J. A. (2007). Intellectual capital management in Spanish universities. Journal of Intellectual Capital, 8(4), 732-748. Doi: https://doi.org/10.1108/14691930710830873

Rendón-Macías, M. E., Villasís-Keever, M. Á. y Miranda-Novales, M. G. (2016). Estadística descriptiva. Revista Alergia México, 63(4), 397-407. Recuperado de http://web.a.ebscohost.com/ehost/detail/detail?vid=0\&sid=4a6bd81d-19e6-488a-b6e04973277ca830\%40sdc-vsessmgr02\&bdata=JnNpdGU9ZWhvc3QtbGl2ZQ\%3d\%3d\#AN=119485428\&db=lth

Richard, P. J., Devinney, T. M., Yip, G. S. and Johnson, G. (2009). Measuring organizational performance: Towards methodological best practice. Journal of Management, 35(3), 718804. Doi: $10.1177 / 0149206308330560$

Robles, J. R. y Zárate, R. E. (2013). Impacto del capital intelectual en facultades de negocios de las universidades públicas. Sotavento M.B.A, 68-81. Recuperado de https://revistas.uexternado.edu.co/index.php/sotavento/article/download/3705/3837/

Rodrigues, C., Selig, P. M. and Viegas, C. V. (2019). A proposal of intellectual capital maturity model (ICMM) evaluation. Journal of Intellectual Capital, 20(2), 208-234. Doi: 10.1108/JIC-12-2016-0130

Rodrigues, H. M., Figueroa, P. F. y Fernández-Jardón, C. M. (2009). La influencia del capital intelectual en la capacidad de innovación de las empresas del sector de automoción de la eurorregión Galicia norte de Portugal. Vigo: Universidad de Vigo. Recuperado de https://www.researchgate.net/profile/Helena_Santos-

Rodrigues/publication/216576193_La_influencia_del_capital_intelectual_en_la_capacida d_de_innovacion_de_las_empresas_del_sector_de_automocion_de_la_eurorregion_Galiz ia_Norte_de_Portugal/links/016a4d1ab47b1

Rodríguez, V., Ortega-Fernández, F., Alvarez, V. and Roqueñí, N. (2006). An Integrated Framework for Intellectual Capital Measurement and Knowledge Management Implementation in Small and Medium-Sized Enterprises. Journal of Information Science, 32(6), 525-538. Doi: 10.1177/0165551506067127

Roos, J., Roos, G., Dragonetti, N. and Edvinsson, L. (1997). Intellectual Capital: Navigating in the new business landscape. New York: New York University Press. 


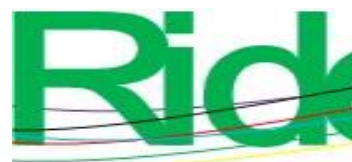

Revista Iberoamericana para la Investigación y el Desarrollo Educativo

ISSN $2007-7467$

Saeed, R., Sami, A., Lodhi, R. N. and Iqbal, A. (2013). Intellectual Capital and Organizational Performance: An Empirical Study in Telecom Sector of Pakistan. Middle-East Journal of Scientific Research, 18(4), 517-523. Doi: 10.5829/idosi.mejsr.2013.18.4.11716

Sánchez, M. P., Elena, S. and Castrillo, R. (2009). Intellectual capital dynamics in universities: a reporting model. Journal of Intellectual Capital, 10(2), 307-324. Doi: $10.1108 / 14691930910952687$

Shehzad, U., Fareed, Z., Zulfiqar, B., Shahzad, F. and Latif, H. S. (2014). The Impact of Intellectual Capital on the Performance of Universities. European Journal of Contemporary Education, 10(4), 237-280. Doi: 10.13187/ejced.2014.10.273

Smart, J. C. y Hamm, R. E. (1993). Organizational effectiveness and mission orientations of twoyear colleges. Research in Higher Education, 34(4), 489-502. Doi: https://doi.org/10.1007/BF00991856

Suraj, O. A. and Bontis, N. (2012). Managing intellectual capital in Nigerian telecommunications companies. Journal of Intellectual Capital, 13(2), 262-282. Doi: https://doi.org/10.1108/14691931211225724

Todericiu, R. y Serban, A. (2015). Intellectual Capital andits Relationship with Universities. Procedia Economics and Finance, 27, 713-717. Doi: https://doi.org/10.1016/S2212$5671(15) 01052-7$

Tseng, C. Y. and Goo, Y. J. (2005). Intellectual capital and corporate value in an emerging economy: empirical study of Taiwanese manufacturers. $R \& D$ Management, 35(2), 187 201. Doi: https://doi.org/10.1111/j.1467-9310.2005.00382.x

Vasconcelo, R., Pedraza, N. A., Lavín, J. y Cortés, A. (2016). La relación del capital intelectual y el desempeño organizacional. Una revisión bibliometrica al 2015. Ideas Concyteg, 11(142). Recuperado de http://sices.guanajuato.gob.mx/resources/ideas/ebooks/142/descargas.pdf Wang, Z., Wang, N. and Liang, H. (2014). Knowledge sharing, intellectual capital and firm performance. Management Decision, 52(2), 230-258. Doi: 10.1108/MD-02-2013-0064

Wee, J. C. and Chua, A. Y. (2015). The Communication of Intellectual Capital-Prevalence and Relationship with Organizational Performance. The Electronic Journal of Knowledge Management, 13(1), 38-50. Retrieved from http://www.ejkm.com/volume13/issue1

Wernerfelt, B. (1984). A Resource-Based View of the Firm. Strategic Management Journal, 5(2), 171-180. Retrieved from https://www.jstor.org/stable/2486175 


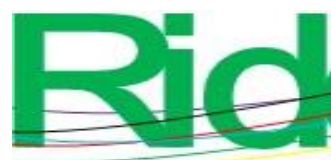

Revista Iberoamericana para la Investigación y el Desarrollo Educativo ISSN 2007 - 7467

Zorrilla, A. (2010). El capital intelectual y su administración en las universidades públicas. XIV Congreso Internacional de la Academia de Ciencias Administrativas, A. C. Monterrey. Recuperado de http://acacia.org.mx/busqueda/pdf/EL_CAPITAL_INTELECTUAL.pdf 